\title{
12. MESOZOIC FORAMINIFERA, LEG 15, DEEP SEA DRILLING PROJECT
}

Emile A. Pessagno, Jr. and Jose F. Longoria T., Geosciences Division, University of Texas, Dallas, Texas

This report lists foraminifers identified in Leg 15 samples and gives a biostratigraphic determination where possible. Only samples with biostratigraphically significant microfossils are listed. Significant radiolarians are also included.

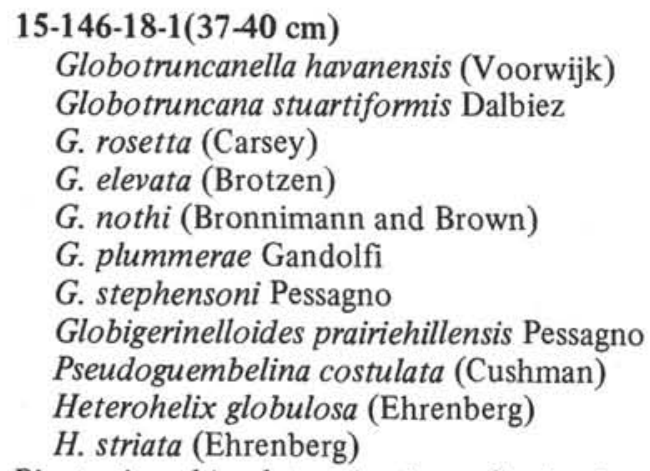

Biostratigraphic determination: G. fornicata-stuartiformis Assemblage Zone, G. elevata Subzone to R. subcircumnodifer Subzone; late Campanian-early Maestrichtian.

15-146-18-3(36-39 cm)

Heterohelix globulosa (Ehrenberg)

Pseudoguembelina costulata (Cushman)

Biostratigraphic determination: Late Campanian-Maestrichtian.

$15-146-18-4(133-137 \mathrm{~cm})$ Globotruncana arca (Cushman)

Biostratigraphic determination: Campanian-Maestrichtian.

15-146-18-5(146-150 cm)

Globotruncanella havanensis (Voorwijk)

Globotruncana stuartiformis Dalbiez

G. rosetta (Carsey)

G. plummerae Gandolfi

G. elevata (Brotzen)

$G$. ventricosa White

$G$. hilli Pessagno

G. fornicata Plummer

G. lapparenti Brotzen

G. bulloides Vogler

Globigerinelloides volutus (White)

Schackoina sp. aff. S. multispinata (Cushman and Wickenden)

Pseudoguembelina costulata (Cushman)

Heterohelix striata (Ehrenberg)

H. globulosa (Ehrenberg)

H. punctulata (Cushman)

Radiolaria: Pseudoaulophacus lenticulatus (White), Dictyomitra multicostata Zittel group.

Biostratigraphic determination: $G$. fornicata-stuartiformis Zone, G. elevata Subzone, G. calcarata Zonule; late Campanian.
$15-146-19-1(137-140 \mathrm{~cm})$

Globotruncana elevata (Brotzen)

G. stuartiformis Dalbiez

G. arca (Cushman)

Pseudotextularia elegans (Rzehak)

Biostratigraphic determination: late Campanian-Maestrichtian.

15-146-19-2(113-116 cm)

Globotruncana elevata (Brotzen)

G. fornicata Plummer

G. linneiana (d'Orbigny)

G. lapparenti Brotzen

G. plummerae Gandolfi

G. stuartiformis Dalbiez

G. rosetta (Carsey)

G. stephensoni Pessagno

G. ventricosa White

G. arca (Cushman)

G. hilli Pessagno?

G. nothi (Bronnimann and Brown)

Globotruncanella sp.

Pseudoguembelina costulata (Cushman)

Biostratigraphic determination: G. fornicata-stuartiformis Assemblage Zone, G. elevata Subzone, G. calcarata Zonule; late Campanian.

15-146-19-3(120-124 cm)

Globotruncana arca (Cushman)

Biostratigraphic determination: Campanian-Maestrichtian.

15-146-20-2(113-118 cm)

Globotruncanella sp.

Heterohelix globulosa (Ehrenberg)

H. pulchra Brotzen

15-146-20-3(108-113 cm)

Globotruncana fornicata Plummer

G. stephensoni Pessagno

G. stuartiformis Dalbiez

G. elevata Brotzen

Pseudoguembelina costulata (Cushman)

Biostratigraphic determination: G. fornicata-stuartiformis Assemblage Zone, G. elevata Subzone to R. subcircumnodifer Subzone; late Campanian-early Maestrichtian.

15-146-22-2(110-113 cm)

Globotruncana calcarata Cushman

Biostratigraphic determination: G. fornicata-stuartiformis Assemblage Zone, G. elevata Subzone, G. calcarata Zonule; late Campanian.

$15-146-23-1(65-70 \mathrm{~cm})$

Globotruncana lapparenti (Brotzen)

G. linneiana (d'Orbigny)

G. elevata (Brotzen) 
Biostratigraphic determination: Late Campanian-early Maestrichtian.

15-146-24-1(127-130 cm)

Radiolaria: Dictyomitra multicostata Zittel group.

Biostratigraphic determination: Turonian-Maestrichtian.

15-146-26-2(127-130 cm)

Radiolaria: Pseudoaulophacus parqueraensis Pessagno, Alievium murphyi Pessagno, A. gallowayi (White) ?, Dictyomitra multicostata Zittel group.

Biostratigraphic determination: Coniacian-late Campanian.

15-146-27-1 $(19-23 \mathrm{~cm})$

Radiolaria: Dictyomitra multicostata Zittel group, Pseudoaulophacus lenticulatus (White).

Biostratigraphic determination: Coniacian-late Campanian.

15-146-28-2(85-87 cm)

Radiolaria: Alievium gallowayi (White) ?, A. murphyi Pessagno, Pseudoaulophacus parqueraensis Pessagno, $P$. lenticulatus (White).

Biostratigraphic determination: Coniacian/SantonianCampanian.

$15-146-30-1(130-132 \mathrm{~cm})$

Radiolaria: Archaeospongoprunum bipartitum Pessagno, $\mathrm{n}$. sp., Patulibracchium teslaensis Pessagno, Dictyomitra multicostata Zittel group, Alievium murphyi Pessagno, A. gallowayi (White), Pseudoaulophacus lenticulatus (White), P. parqueraensis Pessagno, P. venadoensis Pessagno, $P$. praefloresensis Pessagno, $P$. floresensis Pessagno, Crucella espartoensis Pessagno, Conocaryomma lentis Lipman.

Biostratigraphic determination: Santonian (probably late Santonian).

$15-146-32-1(130-134 \mathrm{~cm})$

Same radiolarian assemblage as 15-146-30-1.

15-150-9-1 $(87-89 \mathrm{~cm})$

Marginotruncana concavata (Brotzen)

Biostratigraphic determination: $G$. bulloides Assemblage Zone, $M$. concavata Subzone; early Santonian.

15-151-12-1(104-106 cm)

Globigerinelloides ehrenbergi Barr

Heterohelix striata (Ehrenberg)

Archaeoglobigerina sp. cf. A. blowi Pessagno

A. cretacea (d'Orbigny)

Globotruncana lapparenti Brotzen

G. fornicata Plummer

Marginotruncana marginata (Reuss)

M. pseudolinneiana Pessagno

Biostratigraphic determination: G. bulloides Assemblage Zone, $M$. concavata Subzone (upper part); early Santonian.

$15-151-12-3(73-75 \mathrm{~cm})$

Marginotruncana concavata (Brotzen)

$M$. n. sp.

Archaeoglobigerina cretacea (d'Orbigny)

Heterohelix striata (Ehrenberg)

Globotruncana fornicata Plummer

G. lapparenti Brotzen

Biostratigraphic determination: G. bulloides Assemblage Zone, $M$. concavata Subzone (upper part); early Santonian.
15-152-14-1(100-102 cm)

Globotruncana gansseri Bolli

G. contusa (Cushman) group

G. trinidadensis Gandolfi

G. aegyptiaca Nakkady

G. elevata (Brotzen)

Rugoglobigerina rugosa Plummer

$R$. rotundata Bronnimann

Pseudoguembelina costulata (Cushman)

P. excolata (Cushman)

P. kempensis Esker

Biostratigraphic determination: G. contusa-stuartiformis Assemblage Zone, G. gansseri Subzone (probably upper part); middle Maestrichtian.

15-152-16-1(146-149 cm)

Globotruncana fornicata Plummer

G. lapparenti ss. Brotzen

G. stuartiformis Dalbiez

$G$. n. sp. aff. G. ventricosa White

G. plummerae Gandolfi

$G$. ventricosa White

Rugoglobigerina rugosa Plummer

Heterohelix punctulata (Cushman)

Pseudoguembelina costulata (Cushman)

Biostratigraphic determination: G. fornicata-stuartiformis Assemblage Zone, G. elevata Subzone (undifferentiated); late Campanian.

15-152-16-2(91-94 cm)

Globotruncana fornicata Plummer

G. plummerae Gandolfi

G. rosetta (Carsey)

G. lapparenti Brotzen

G. stuartiformis Dalbiez

G. elevata (Brotzen)

G. stephensoni Pessagno

G. bulloides Vogler

$G$. n. sp. aff. G. ventricosa White

Rugotruncana subcircumnodifer (Gandolfi)

Biostratigraphic determination: $G$. formicata-stuartiformis Assemblage Zone, G. elevata Subzone (undifferentiated); late Campanian.

15-152-16-2(91-94 cm)

Globotruncana fornicata Plummer

G. plummerae Gandolfi

G. rosetta (Carsey)

G. lapparenti Brotzen

G. stuartiformis Dalbiez

G. elevata (Brotzen)

G. stephensoni Pessagno

G. bulloides Vogler

$G$. n. sp. aff. G. ventricosa White

Rugotruncana subcircumnodifer (Gandolfi)

Biostratigraphic determination: G. fornicata-stuartiformis Assemblage Zone, G. elevata Subzone, G. calcarata Zonule (via superposition); latest Campanian.

$15-152-17-1(80-85 \mathrm{~cm})$

Globotruncana elevata Brotzen

G. stuartiformis Dalbiez

G. arca (Cushman)

G. lapparenti Brotzen

G. stephensoni Pessagno 
G. fornicata Plummer

$G$. n. sp. aff. $G$. ventricosa White

Rugotruncana subcircumnodifer (Gandolfi)

Heterohelix striata (Ehrenberg)

G. punctulata (Cushman)

Pseudoguembelina costulata (Cushman)

Biostratigraphic determination: $G$. formicata-stuartiformis Assemblage Zone, G. elevata Subzone, G. calcarata Zonule (via superposition); latest Campanian.

$15-152-18-1(68-70 \mathrm{~cm})$

Globotruncana elevata Brotzen

$G$. ventricosa White

G. fornicata Plummer

G. stuartiformis Dalbiez

G. rosetta (Carsey)

G. linneiana (d'Orbigny)

Pseudoguembelina costulata (Cushman)

Rugoglobigerina sp.

Biostratigraphic determination: G. fornicata-stuartiformis Assemblage Zone, G. elevata Subzone (undifferentiated); late Campanian.

15-152-18-2(141-144 cm)

Globotruncana subspinosa Pessagno

$G$. ventricosa White

G. fornicata Plummer

G. stuartiformis Dalbiez

G. plummerae Gandolfi

G. stephensoni Pessagno

$G$. sp. aff. G. ventricosa White

Rugoglobigerina rugosa (Plummer)

Pseudoguembelina costulata (Cushman)

Heterohelix punctulata (Cushman)

Globigerinelloides sp. aff. G. yaucoensis (Pessagno)

Biostratigraphic determination: $G$. fornicata-stuartiformis Assemblage Zone, G. elevata Subzone; late Campanian.

$15-152-19-1(103-106 \mathrm{~cm})$

Globotruncana calcarata Cushman

$G$. n. sp. aff. $G$. ventricosa White

G. fornicata Plummer

G. elevata (Brotzen)

G. subspinosa Pessagno

G. hilli Pessagno

G. lapparenti Brotzen

Archaeoglobigerina cretacea (d'Orbigny)

Rugoglobigerina rugosa (Plummer)

Globigerinelloides yaucoensis (Pessagno)

G. prairiehillensis Pessagno

Pseudoguembelina costulata (Cushman)

Heterohelix striata (Ehrenberg)

H. globulosa (Ehrenberg)

H. sp.

Biostratigraphic determination: G. fornicata-stuartiformis Assemblage Zone, G. elevata Subzone, G. calcarata Zonule; latest Campanian.

\section{$15-152-21-1(108-114 \mathrm{~cm})$}

Same assemblage as 15-152-19-1(103-106 cm) with Globotruncana calcarata (Cushman). Biostratigraphic determination: latest Campanian.

$15-153-12-2(75-78 \mathrm{~cm})$

Globotruncana bulloides Vogler

G. elevata Brotzen
Pseudoguembelina excolata (Cushman)

Pseudotextularia elegans Rzehak

Biostratigraphic determination: G. fornicata-stuartiformis Assemblage Zone, R. subcircumnodifer Subzone; early Maestrichtian.

\section{5-153-12-3}

Globotruncana fornicata Plummer

G. plummerae Gandolfi

Pseudotextularia elegans Rzehak

Biostratigraphic determination: G. fornicata-stuartiformis Assemblage Zone (undifferentiated); Campanian-early Maestrichtian.

15-153-13-2(28-30 cm)

Globotruncana lapparenti s.s. Brotzen

G. linneiana (d'Orbigny)

G. fornicata Plummer

G. rosetta (Carsey)

G. hilli Pessagno

G. stuartiformis Dalbiez

G. elevata Brotzen

Rugoglobigerina rugosa Plummer

Globigerinelloides yaucoensis (Pessagno)

G. prairiehillensis Pessagno

Pseudoguembelina costulata (Cushman)

Heterohelix punctulata (Cushman)

Biostratigraphic determination: G. fornicata-stuartiformis Assemblage Zone, G. elevata Subzone, G. calcarata Zonule to $R$. subcircumnodifer Subzone, G. lapparenti Zonule; late Campanian-early Maestrichtian.

15-153-13-4(58-60 cm)

Globotruncanella havanensis (Voorwijk)

Globotruncana arca (Cushman)

G. stephensoni (Pessagno)

G. stuartiformis Dalbiez

G. rosetta (Carsey)

G. fornicata Plummer

Pseudoguembelina costulata (Cushman)

Heterohelix striata (Ehrenberg)

Rugoglobigerina rugosa (Plummer)

Biostratigraphic determination: G. fornicata-stuartiformis Assemblage Zone, G. elevata Subzone to $R$. subcircumnodifer Subzone; late Campanian-early Maestrichtian.

$15-153-14-1(97-100 \mathrm{~cm})$

Globotruncana fornicata Plummer

G. arca (Cushman)

G. linneiana (d'Orbigny)

G. elevata (Brotzen)

G. stuartiformis Dalbiez

G. stephensoni Pessagno

G. bulloides (Vogler)

Rugoglobigerina rugosa Plummer

Globigerinelloides yaucoensis (Pessagno)

G. volutus (White)

Heterohelix striata (Ehrenberg)

Pseudoguembelina costulata (Cushman)

Biostratigraphic determination: G. fornicata-stuartiformis Assemblage Zone, G. elevata Subzone, G. calcarata Zonule to $R$. subcircumnodifer Subzone (undifferentiated); late Campanian-early Maestrichtian. 
E. A. PESSAGNO, JR., J. F. LONGORIA, T.

\section{$15-153-16-2(70-72 \mathrm{~cm})$}

\author{
Marginotruncana concavata (Brotzen) \\ $M$. angusticarenata (Gandolfi) \\ M. coronata (Bolli) \\ $M$. indica (Jacob and Sastry) \\ $M$. marginata (Reuss)
}

Globotruncana fornicata Plummer

Whiteinella archeocretacea Pessagno

Loeblichella sp.

Heterohelix reussi (Cushman)

Biostratigraphic determination: $G$. bulloides Assemblage Zone, $M$. concavata Subzone (upper part); early Santonian. 